\title{
Standardization of the Japanese version of the Glasgow Sensory Questionnaire (GSQ)
}

Yuko Takayama ${ }^{\text {a }}$, Ryuichiro Hashimoto a,b,c ${ }^{\text {, Masayuki Tani }}{ }^{\text {a }}$, Chieko Kanai ${ }^{\text {a }}$, Takashi Yamada $^{\text {a }}$, Hiromi Watanabe ${ }^{\text {a }}$, Taisei Ono ${ }^{\text {a }}$, Nobumasa Kato ${ }^{\text {a,c }}$, Akira Iwanami ${ }^{\text {a }}$

${ }^{a}$ Department of Psychiatry, Showa University, Karasuyama Hospital, 6-11-11

Kitakarasuyama, Setagayaku, Tokyo 157-8577, Japan

${ }^{\mathrm{b}}$ Department of Language Sciences, Graduate School of Humanities, Tokyo Metropolitan University

1-1 Minami Osawa, Hachioji-shi, Tokyo, 192-0364 Japan

${ }^{\mathrm{c}}$ Japan Science and Technology Agency, CREST, Tokyo, Japan

Corresponding author: Ryuichiro Hashimoto, Ph.D., Department of Psychiatry, Showa University, Karasuyama Hospital, 6-11-11 Kitakarasuyama, Setagayaku, Tokyo 157-8577, Japan. Tel: +81333005231

Fax: +8133308 1710

E-mail: dbridges50@gmail.com (R. Hashimoto),

\begin{abstract}
Individuals with autism spectrum disorders (ASD) often have sensory processing abnormalities. However, limited measures that assess these problems in adults with ASD have been developed till date, particularly in Japan. Robertson and Simmons (2012) developed a self-rating scale to investigate sensory sensitivity: the Glasgow Sensory Questionnaire (GSQ). In the present study, we developed a Japanese version of GSQ and investigated sensory abnormalities in adults with ASD. We compared results of the Japanese
\end{abstract}


version of GSQ in adults between an ASD group $(n=64)$ and a control group $(n=70)$. In addition, we also administered these individuals with the Autism Spectrum Quotient (AQ), which is a questionnaire for assessing autistic traits. The Japanese version of GSQ scores were significantly higher in the ASD group than that in the control group. The total GSQ score and each sensory subscale showed a positive correlation with AQ in the total study sample. These results indicate that individuals with pronounced autistic traits have more frequent and extreme sensory processing problems compared with that in individuals with less pronounced autistic traits. We also assessed validity of the new test. Cronbach's $\alpha$ of the questionnaire was calculated, and its high value indicates that the Japanese version of GSQ has high reliability.

Key words: Autism Spectrum Disorders; Sensory sensitivity; Glasgow Sensory Questionnaire; Autism Spectrum Quotient 


\section{Introduction}

Autism spectrum disorders (ASD) also known as pervasive developmental disorders (PDD) are characterized by markedly abnormal or impaired development of social interaction and a restricted and stereotyped repertoire of activities and interests (American Psychiatric Association, 1994, 2013; World Health Organization, 1993). According to previous studies, individuals with ASD often have sensory processing problems. Crane et al. (2009) showed that $94.4 \%$ of adults with ASD have extreme levels of sensory processing problems in at least one sensory domain. In each sensory domain, such as visual (Simmons et al., 2009), auditory (Haesen et al., 2011), and tactile (Cascio et al., 2008), various studies reported differences between the ASD and control groups.

Most problems of sensory modulation in ASD are expressed as either hyper- and/or hyposensitivity (Rogers, Hepburn, \& Wehner, 2003). An individual with hypersensitivity is uncomfortable with or avoids a particular sensory stimulus that most people do not perceive or regard important because of weakness of the stimuli. In contrast, an individual with hyposensitivity either reacts too weakly to a particular sensory stimulus or actively pursues the stimulus (Ben-Sasson et al., 2007). Previous studies reported that the sensory modulation problem in ASD is associated with symptoms of anxiety (Green, Ben-Sasson, Soto, \& Cater, 2012) and social isolation (Cosbey, Johnston, \& Dunn, 2010).

The sensory modulation problems in ASD have not been included in the 3 concepts of ASD proposed by Wing (1996) and in the universal standard diagnostic criteria: the Diagnostic and Statistical Manual of Mental Disorders (DSM-IV; American Psychiatric Association, 1994) and the International Classification of Diseases (ICD-10; World Health Organization, 1993). However, in 2013, those problems were finally acknowledged in a new edition of the Diagnostic and Statistical Manual of Mental Disorders (DSM-5; American Psychiatric Association, 2013), and thereafter, the sensory modulation problems have gained 
recognition as one of the core symptoms of ASD. DSM-5 describes "hyper- or hyporeactivity to sensory input or unusual interest in sensory aspects of the environment" as a symptomatic criteria of ASD. Understanding of sensory features of ASD is important and necessary for diagnosis, assessment of comorbid conditions, and clinical interventions for sensory symptoms and social adjustment.

On the other hand, there are some problems in assessment of the sensory features of ASD because existing standard clinical tests do not necessarily detect these sensory processing problems (Simmons et al., 2009). The need for tests that can detect sensory processing problems in the daily life of individuals with ASD has resulted in the development of several rating scales using the English language in the past; however, such scales are not available in Japanese. Brown and Dunn (2002) published a self-report sensory questionnaire (the Adult/Adolescent Sensory Profile; AASP), and it is currently being standardized in Japanese (Ito et al., 2013). Although this rating scale is valid and effective, it was designed to quantify sensory problems in various mental and physical disorders, not specifically in individuals with ASD.

Recently, Robertson and Simmons (2012) developed a self-rating sensory scale, the Glasgow Sensory Questionnaire (GSQ). Because individuals with ASD are located at the extreme end of a continuum of autistic traits that are also present in the general population (Constantino \& Todd, 2003), the researchers tested GSQ on the general population and compared the results with the Autism Spectrum Quotient (AQ; Baron-Cohen et al., 2001). Their data suggest a strong link between sensory processing and autistic traits in the general population.

We decided that developing a Japanese version of GSQ was a worthwhile task for evaluating sensory abnormalities in individuals with ASD in Japan; therefore, we developed and validated this GSQ and investigated sensory problems in Japanese individuals with ASD. 


\section{Methods}

\subsection{Participants and procedures}

Participating patients and healthy individuals provided written consent prior to administration of the questionnaires and subsequent testing. The study protocol was approved by the Ethics Committee of the Faculty of Medicine of Showa University.

The clinical group of the study comprised 64 outpatients from the Showa University Hospital (mean age, 30.4 years; range, 20-48 years; standard deviation $(\mathrm{SD})=6.6$; 56 men and 8 women). All patients were referred to our institution by physicians from other clinics. Inclusion criteria were age of 18-60 years and a formal diagnosis of PDD on the basis of DSM-IV criteria. Exclusion criteria were comorbid psychiatric disorders according to DSM-IV Axis I or II and presence of a neurological disease or severe medical illness. A team of 2 experienced psychiatrists and a clinical psychologist performed the diagnostic assessment. In this study, PDD included Autistic disorder, Asperger's disorder and Pervasive developmental disorder, not otherwise specified. Intelligence was assessed using either the Wechsler Adult Intelligence Scale tests (WAIS-R, Wechsler, 1981; WAIS-III, Wechsler, 1997) or the Japanese version of the National Adult Reading Test (Nelson \& Willison, 1991; Matsuoka, Uno, Kasai, Koyama, \& Kim, 2006). In the intelligence test there were 58 participants with a mean intelligence quotient (IQ) of 102.5 (range, 71-135; SD = 15.6). Although 6 participants have not been assessed till date, we confirmed adequacy of their intelligence with the help of their schooling history and information from their primary physicians.

The assessment included detailed interviews with the patients to analyze their development and behavior from infancy to adolescence (developmental history, present illness, and past medical history) and family history. The interviews were performed independently by 2 psychiatrists and a clinical psychologist. The patients were also asked to 
bring suitable informants who knew the patient in early childhood. At the end of the clinical interview, the patients were diagnosed according to the DSM-IV criteria for PDD based on consensus among the psychiatrists and the clinical psychologist. Patients diagnosed with PDD were asked by the psychiatrists to complete AQ and the Japanese version of GSQ.

The control group included 70 adults, who were recruited by announcements and via acquaintances through Showa University Hospital (mean age, 31.1 years; range, 20-46 years; $\mathrm{SD}=6.6 ; 55$ men and 15 women). None of the control subjects had an Axis I psychiatric disorder, neurological disease, or severe medical illness. All participants volunteered to participate in the study after receiving an adequate explanation regarding the study. The control subjects were asked to complete AQ and the Japanese version of GSQ. The ASD and control groups did not differ significantly in age $(\mathrm{t}=0.635, \mathrm{df}=132, \mathrm{p}=0.527)$ or sex ratio $\left(\chi^{2}=1.875, p=0.171\right)$.

\subsection{Instruments}

\subsubsection{The Glasgow Sensory Questionnaire}

This test was developed by Robertson and Simmons (2012) and is a 42-item self-administered questionnaire for adults for detection of either hyper- or hyposensitivity in 7 domains: visual, auditory, gustatory, olfactory, tactile, vestibular, and proprioceptive. The test items were equally distributed among the sensory domains, with 3 questions assessing possible hypersensitivity and 3 testing hyposensitivity. All items questioned how frequently certain sensory events were experienced, on the following scale: never, rarely, sometimes, often, and always. Responses were coded on a scale from 0 to 4, with possible total scores ranging from 0 to 168. Robertson and Simmons (2012) reported a highly significant positive correlation between the GSQ score and the number of autistic traits as assessed by AQ in adult general population. 


\subsubsection{The Autism Spectrum Quotient}

This test was developed by Baron-Cohen, Wheelwright, Skinner, Martin, and Clubley (2001) and is a 50-item self-administered questionnaire for adults with normal intelligence for detection of autistic traits. The 50 items were equally distributed among 5 domains: social skills, attention switching, attention to detail, communication, and imagination. Each AQ item is a brief statement followed by 4 possible ratings: definitely agree, slightly agree, slightly disagree, and definitely disagree. However, each item is scored as either 0 or 1 and the highest total score is 50. A higher score indicates more autistic traits. Baron-Cohen et al. (2001) showed that cutoff score of 32 discriminates between individuals with Asperger's disorder/high-functioning autism and normal controls. The Japanese version of AQ was standardized for use in Japan (Wakabayashi, Baron-Cohen, \& Wheelwright, 2006), which is reported to have good internal consistency (Cronbach's $\alpha>0.70$ ), test-retest reliability, and discriminant validity (Kurita, Koyama, \& Osada, 2005; Wakabayashi, Baron-Cohen, Wheelwright, \& Tojo, 2006). Wakabayashi et al. (2006) reported that a cutoff of 33 discriminates between individuals with Asperger's disorder/high-functioning autism and normal controls. Internal consistency of AQ in the present study was high in the ASD (Cronbach's $\alpha=0.83$ ) and control groups (Cronbach's $\alpha=0.83$ ).

\subsection{Translation of the test items}

Under an agreement with the authors (Robertson and Simmons at the University of Glasgow), the 42 items of GSQ were translated into Japanese by 3 investigators. The preliminary Japanese translation was then back-translated by a bilingual Japanese person and an English speaker who is fluent in Japanese. Both of them were not given any information about the original English version of GSQ. The back-translation had the same 
multiple-choice format and was sent to the authors to confirm its equivalence to the original English version. They reviewed the first back-translation and made comments on some problematic items. We repeated the translation and back-translation procedures until the publisher and authors confirmed the equivalence of English and Japanese versions. The final approved version of the translation was used in the present study.

\subsection{Statistical analysis}

We compared age and the scores on AQ and GSQ between the ASD and control groups using the independent samples $t$ test. We compared the sex ratio of the 2 groups using Pearson's $\chi^{2}$ test. We examined the relationships between the scores on AQ and GSQ using Pearson's correlation coefficient, where $\mathrm{p}$ value of $<0.05$ (2-tailed test) was considered statistically significant. Cronbach's $\alpha$ was calculated to examine the internal consistency of the tests. We performed all calculations using the SPSS software, version 20.0 (SPSS, Tokyo, Japan).

\section{Results}

\subsection{Test scores}

The scores on AQ, the Japanese version of GSQ, and their respective subscales are listed in Table 1. The mean total score of AQ was significantly higher in the ASD group than that in the control group $(\mathrm{t}=13.957, \mathrm{df}=131, \mathrm{p}<0.001)$ and, the score of each subscale of AQ was significantly higher in the ASD group than that in the control group. The mean total score on the Japanese version of GSQ was also significantly higher in the ASD group than that in the control group $(\mathrm{t}=4.872, \mathrm{df}=132, \mathrm{p}<0.001)$ and, the score on each sensory domain except olfactory was significantly higher in the ASD group than that in the control group. 


\subsection{The relationship between the sensory scores and AQ scores}

The total sensory score showed a significant positive correlation with the total AQ score in the ASD group $(r=0.553, p<0.001)$, in the control group $(r=0.559, \mathrm{p}<0.001)$, and in the total study sample $(r=0.626, p<0.001)$. The relationship between the total sensory and the total AQ score is shown in Fig. 1. These data indicated that individuals with high AQ scores reported having more frequent and extreme reactions (either hyper- or hyposensitivity) to sensory stimuli compared with that in individuals with lower AQ scores, regardless of the presence of a diagnosis of ASD. The correlations were calculated for each subscale of AQ in the total study population. The data from all of the subscales indicated a significant positive correlation with the total sensory score (Table 2). We also analyzed each sensory domain in the total study population. The score from each domain had a positive linear relationship with the total AQ score, including the olfactory domain (Table 3).

\section{[Insert Figure 1 here]}

[Insert Table 2 here]

[Insert Table 3 here]

\subsection{Internal consistency}

The internal consistency of the Japanese version of GSQ was supported by Cronbach's $\alpha$ values of 0.920 for the total study population, 0.925 for the ASD group, and 0.893 for the control group. The exclusion of any probable inconsistent item from the analysis will increase $\alpha$ value even higher. However, every item raised the $\alpha$ value by $<0.01$, when excluded from 
the analysis.

\section{Discussion}

In the present study, we developed and validated a Japanese version of GSQ, which is a self-rating test for measuring sensory sensitivity. We investigated characteristic features of sensory processing in adult with ASD in comparison with a control group. The results were highly comparable to those of the original GSQ study. The present study also assessed scientific validity of the new test by analyzing the adequate number of mean value of Cronbach's $\alpha$ high reliability and internal consistency of the Japanese version of GSQ.

In this study, the scores of the Japanese version of GSQ were significantly higher in the ASD group than that in the control group. The total GSQ score and each sensory subscale shows a positive correlation with $\mathrm{AQ}$, which is a validated self-rating test for detection of autistic traits. These results indicate that individuals with pronounced autistic traits have more frequent and extreme reactions (either hyper- or hyposensitivity) to sensory stimuli compared with individuals with less pronounced autistic traits, with or without the diagnosis of ASD. Robertson and Simmons (2012) reported the same conclusion after comparing the original GSQ with AQ in the general population (age range, 16-66 years). We also showed a significant correlation between the sensory ability and autistic traits in a broad sample that includes both general population and individuals with ASD. Our results suggest that the sensory processing problem of ASD is also noted in the general population.

In subscales of the Japanese version of GSQ, each sensory domain (visual, auditory, gustatory, olfactory, tactile, vestibular, and proprioceptive) had a significant correlation with AQ. These data indicate that there is a tendency for individuals with autistic traits to have sensory problems in multiple sensory domains. In contrast, the score in each sensory domain except olfactory was significantly higher in the ASD group than that in the control group. 
According to our results, olfactory problems may be more likely to manifest themselves in autistic patients, but less frequently as to reach statistical significance. There have been only a few studies that examined olfactory problems in individuals with ASD. Some studies reported that sensitivity (Dudova et al., 2011) and the identification ability (Bennetto, Kuschner, \& Hyman, 2007) are diminished in individuals with ASD, but Tavassoli and Baron-Cohen (2011) reported no difference between individuals with ASD and normal controls in the olfactory domain. Therefore, further research is needed in this area.

Sensory processing problems in ASD have been widely reported. A meta-analysis indicates that at least $50 \%$ of each study population reported a sensory abnormality (Ben-Sasson et al., 2009). However, majority of the research in this area has focused on children. Crane et al. (2007) showed that $94.4 \%$ of adults with ASD have extreme levels of sensory processing problems (low registration, sensation seeking, sensory sensitivity, or sensory avoidance). Nevertheless, there has been no structured research on which sensory domain has problems with a higher frequency of abnormalities or a higher degree of severity. Pathophysiology of sensory processing problems in ASD has also not been elucidated till date. A number of authors have suggested that the signal-to-noise ratio in the neural networks may be higher in individuals with ASD, and this anomaly may be related to sensory problems in ASD (reviewed by Simmons et al., 2009). Some studies have reported that the neural noise in autism is a result of abnormal brain connectivity in the sensory cortex (reviewed by Belmonte et al., 2004; Wass, 2011).

Simmons and Robertson (2012) showed that sensory sensitivity could affect productivity and ability to concentrate in individuals with ASD. Avoidance of people and places as a result of various types of sensory intolerance could easily lead to social isolation (Cosbey \& Dunn, 2010). The results of the present study suggest that the use of GSQ may improve understanding of the abnormalities in sensory sensitivity. This knowledge may help in 
decreasing the difficulties and distress in the patients' daily and social life and designing a more effective medical intervention by taking into account the sensory processing problems in individuals with ASD. Coordination between environment and medication as well as fine-tuning of occupational therapy and psychological counseling in accordance with a patient's sensory profile should decrease the burden of sensory problems and facilitate daily social- and work-related interactions.

A limitation of the present study is that we were unable to assess IQ in all subjects. A previous study found a modest negative correlation between autistic traits and IQ, which was mainly explained by communication problems (Hoekstra et al., 2010). However, we would like to stress that autistic traits and IQ are largely independent. Rogers, Hepburn, and Wehner (2003) reported that sensory symptoms have no relationship with IQ. We asked participants to disclose whether there was any difficulty with understanding the questionnaire, and no one reported comprehension difficulties.

We expect that our development of the Japanese version of the GSQ will provide a valuable means for assessing sensory abnormalities in the Japanese individuals with ASD. Further validation will be performed using a larger sample size, which will lead to more productive research inquiry in the Japanese population such as testing possible gender differences in sensory processing.

\section{Acknowledgements}

We would like to thank Dr. D. Simmons, A. Robertson for kindly giving us a permission to translate the questionnaire and for helping with the back-translation process. We would also like to thank Drs. M. Kawato and F. Pollick for introducing the original version of the questionnaire. This work was supported by CREST of Japanese Science and Technology (JST) to KN. 


\section{References}

American Psychiatric Association. (1994). Diagnostic and statistical manual of mental disorders (4th ed.). Washington, DC: American Psychiatric Association.

American Psychiatric Association. (2013). Diagnostic and statistical manual of mental disorders (5th ed.). Washington, DC: American Psychiatric Association.

Ban-Sasson, A., Hen, L., Fluss, R., Cermak, S. A., Engel-Yeger, B., \& Gal, E. (2009). A meta-analysis of sensory modulation symptoms in individuals with autism spectrum disorders. Journal of Autism and Developmental Disorders, 39, 1-11.

Baron-Cohen, S., Wheelwright, S., Skinner, R., Martin, J., \& Clubley, E. (2001). The Autism-Spectrum Quotient (AQ): Evidence from Asperger syndrome/high-functioning autism, males and females, scientists and mathematicians. Journal of Autism and Developmental Disorders, 31, 5-17.

Bennetto, L., Kuschner, E. S., \& Hyman, S. L. (2007). Olfaction and taste processing in autism. Biological Psychiatry, 62, 1015-1021.

Ben-Sasson, A., Cermak, S. A., Orsmond, G. I., Tager-Flusberg, H., Carter, A. S., Kadlec, M. B., et al. (2007). Extreme sensory modulation behaviors in toddlers with autism spectrum disorders. The American Journal of Occupational Therapy, 61, 584-592.

Belmonte, K., Allen, G., Beckel-Mitchener, A., Boulanger, L. M., Carper R. A., \& Webb, S. J. (2004). Autism and abnormal development of brain connectivity. The Journal of Neuroscience, 24, 9228-9231.

Brown, C., \& Dunn, W. (2002). Adult/adolescent sensory profile: User's manual. San Antonio: Psychological Corporation.

Brown, C., Tollefson, N., Dunn, W., Cromwell, R., \& Filion, D. (2001). The Adult Sensory Profile: measuring patterns of sensory processing. The American Journal of Occupational Therapy, 55, 75-82.

Cascio, C., McGlone, F., Folger, S., Tannan, V., Baranek, G., Pelphrey, K. A., et al. (2008). Tactile perception in adults with autism: A multidimensional psychophysical study. Journal of Autism and Developmental Disorders, 38, 127-138.

Cosbey, J., Johnston, S. S., \& Dunn, L. (2010). Sensory Processing disorders and social participation. American Journal of Occupational Therapy, 64, 462-473.

Crane, L., Goddard, L., Pring, L. (2009). Sensory processing in adults with autism spectrum disorders. Autism, 13, 215-228.

Dudova I. Vodicka, J., Havlovicova, M., Sedlacek, Z., Urbanek, T., \& Hrdlicka, M. (2011). Odor detection threshold, but not odor identification, is impaired in children with autism. European Child and Adolescent Psychiatry, 20, 333-340.

Green, S. A., Ben-Sasson, A., Soto, T. A., \& Cater, A. S. (2012). Anxiety and sensory over-responsivity in toddlers with autism spectrum disorders: bidirectional effects across 
time. Journal of Autism and Developmental Disorders, 42, 1112-1119.

Haesen, B., Boets, B., \& Wagemans, J. (2011). A review of behavioural and electrophysiological studies on auditory processing and speech perception in autism spectrum disorders. Research in Autism Disorders, 5, 701-714.

Hoekstra, R. A., Happé, F., Baron-Cohen, S., \& Ronald, A. (2010). Limited genetic covariance between autistic traits and intelligence: Findings from a longitudinal twin study. American Journal of Medical Genetics Part B: Neuropsychiatric Genetics, 153, 994-1007.

Ito, H., Hirakawa, T., Hagiwara, T., Iwanaga, R., Tani, I., Yukihiro, R., et al. (2013). Standardization of the Japanese version of the Sensory Profile: Reliability and norms based on a community sample. Seishinigaku, 44, 537-548 (in Japanese).

Kurita, H., Koyama, T., \& Osada, H. (2005). Autism-spectrum Quotient-Japanese version and its short forms for screening normally intelligent persons with pervasive developmental disorders. Psychiatry and Clinical Neurosciences, 4, 490-496.

Matsuoka, K., Uno, M., Kasai, K., Koyama, K., \& Kim, Y. (2006). Estimation of premorbid IQ in individuals with Altzheimer's disease using Japanese ideographic script (Kanji) compound words: Japanese version of National Adult Reading Test. Psychiatry and Clinical Neurosciences, 60, 332-339.

Robertson, A. E., \& Simmons, D. R. (2012). The relationship between sensory sensitivity and autistic traits in the general population. Journal of Autism and Developmental Disorders, 43, 775-784.

Rogers, S. J., Hepburn, S., \& Wehner, E. (2003). Parent reports of sensory symptoms in toddlers with Autism and those with other Developmental disorders. Journal of Autism and Developmental Disorders, 33, 631-642.

Simmons, D. R., \& Robertson, A. E. (2012). Visual symptoms in adults with autism spectrum disorders. i-Perception, 3, 397.

Simmons, D. R., Robertson, A. E., McKay, L. S., Toal, E., McAleer, P., \& Pollick, F. E. (2009). Vision in autism spectrum disorders. Vision Research, 49, 2705-2739.

Tavassoli, T., \& Baron-Cohen, S. (2012). Taste identification in adults with autism spectrum conditions. Journal of autism and Developmental Disorders, 42, 1419-1424.

Wakabayashi, A., Baron-Cohen, S., Wheelwright, S., \& Tojo, Y. (2006). The Autism-Spectrum Quotient (AQ) in Japan: Across-cultural comparison. Journal of Autism and Developmental Disorders, 36, 263-270.

Wass, S. (2011). Distortions and disconnections: Disrupted brain connectivity in autism. Brain and Cognition, 75, 18-28.

Wing, W. (1996). Autistic spectrum disorders. British Medical Journal, 1996, 312.

World Health Organization. (1993). The ICD-10 classification of mental and behavioural disorders: Diagnostic criteria for research. Geneva: WHO. 
Table 1. A description of the AQ and GSQ scores.

\begin{tabular}{|c|c|c|c|c|c|c|c|c|}
\hline & \multicolumn{3}{|c|}{$\operatorname{ASD}(n=64)$} & \multicolumn{3}{|c|}{ Control $(\mathrm{n}=70)$} & \multirow[t]{2}{*}{$\mathrm{t}$ value } & \multirow[t]{2}{*}{$\mathrm{p}$ value } \\
\hline & Mean & SD & Range & Mean & SD & Range & & \\
\hline Age (years) & 30.4 & 6.6 & $20-48$ & 31.1 & 6.6 & $20-46$ & 0.635 & 0.527 \\
\hline \multicolumn{9}{|l|}{$\mathrm{AQ}$} \\
\hline Total (0-50) & 33.8 & 7.2 & $17-47$ & 16.0 & 7.4 & $3-36$ & 13.957 & $<0.001^{\mathrm{a}}$ \\
\hline Social skills & 2.7 & 2.6 & $0-10$ & 7.3 & 2.7 & $0-10$ & 10.263 & $<0.001^{\mathrm{a}}$ \\
\hline $\begin{array}{l}\text { Attention } \\
\text { switching }\end{array}$ & 4.0 & 2.0 & $0-9$ & 7.4 & 2.1 & $2-10$ & 9.270 & $<0.001^{\mathrm{a}}$ \\
\hline $\begin{array}{l}\text { Attention to } \\
\text { detail }\end{array}$ & 3.9 & 2.2 & $0-9$ & 5.6 & 2.1 & $0-9$ & 4.631 & $<0.001^{\mathrm{a}}$ \\
\hline Communication & 2.6 & 2.4 & $0-9$ & 6.8 & 2.1 & $2-10$ & 10.719 & $<0.001^{\mathrm{a}}$ \\
\hline Imagination & 2.9 & 1.6 & $0-7$ & 6.3 & 2.2 & $2-10$ & 10.195 & $<0.001^{\mathrm{a}}$ \\
\hline \multicolumn{9}{|l|}{$\begin{array}{l}\text { GSQ (Japanese } \\
\text { version) }\end{array}$} \\
\hline Total $(0-168)$ & 51.5 & 20.0 & $12-88$ & 35.5 & 18.0 & $5-85$ & 4.872 & $<0.001^{\mathrm{a}}$ \\
\hline Visual & 7.6 & 4.0 & $0-16$ & 5.0 & 3.7 & $0-16$ & 3.829 & $<0.001^{\mathrm{a}}$ \\
\hline Auditory & 12.2 & 4.5 & $2-23$ & 9.7 & 4.0 & $2-20$ & 3.379 & $<0.001^{\mathrm{a}}$ \\
\hline Gustatory & 6.0 & 3.6 & $0-13$ & 3.9 & 2.7 & $0-11$ & 3.829 & $<0.001^{\mathrm{a}}$ \\
\hline Olfactory & 5.6 & 3.6 & $0-16$ & 5.1 & 2.9 & $1-12$ & 0.948 & 0.345 \\
\hline Tactile & 6.3 & 3.7 & $0-13$ & 4.0 & 2.7 & $0-13$ & 3.982 & $<0.001^{\mathrm{a}}$ \\
\hline Vestibular & 6.3 & 3.5 & $0-15$ & 3.8 & 3.0 & $0-11$ & 4.394 & $<0.001^{\mathrm{a}}$ \\
\hline Proprioceptive & 7.7 & 3.8 & $0-16$ & 4.0 & 3.2 & $0-16$ & 6.089 & $<0.001^{\mathrm{a}}$ \\
\hline
\end{tabular}

Legend: AQ = Japanese version of Autism Spectrum Quotient; GSQ = Glasgow Sensory;

Questionnaire; ASD = autism spectrum disorders.

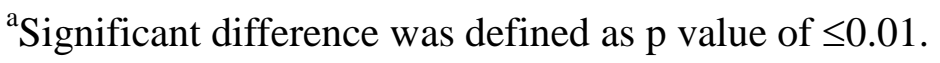


Table 2. Correlations of AQ with the total GSQ score in the total study population.

\begin{tabular}{lcc} 
& \multicolumn{3}{c}{ correlation with } \\
& \multicolumn{2}{c}{ total GSQ score } \\
\cline { 2 - 3 } AQ & r value & p value \\
\hline Total & 0.626 & $<0.001^{\mathrm{a}}$ \\
Social skills & 0.455 & $<0.001^{\mathrm{a}}$ \\
Attention switching & 0.626 & $<0.001^{\mathrm{a}}$ \\
Attention to detail & 0.320 & $<0.001^{\mathrm{a}}$ \\
Communication & 0.650 & $<0.001^{\mathrm{a}}$ \\
Imagination & 0.424 & $<0.001^{\mathrm{a}}$
\end{tabular}

Legend: AQ = Japanese version of Autism Spectrum Quotient; GSQ = Glasgow Sensory Questionnaire.

${ }^{\mathrm{a}}$ Significant correlation among the total GSQ score was defined as $\mathrm{p} \leq 0.01$. 
Table 3. Correlations between GSQ and the total AQ score in the total study population.

correlation with

total AQ score

\begin{tabular}{lcc}
\cline { 2 - 2 } GSQ & r value & p value \\
\hline Total & 0.626 & $<0.001^{\mathrm{a}}$ \\
Visual & 0.484 & $<0.001^{\mathrm{a}}$ \\
Auditory & 0.541 & $<0.001^{\mathrm{a}}$ \\
Gustatory & 0.491 & $<0.001^{\mathrm{a}}$ \\
Olfactory & 0.274 & $0.001^{\mathrm{a}}$ \\
Tactile & 0.504 & $<0.001^{\mathrm{a}}$ \\
Vestibular & 0.546 & $<0.001^{\mathrm{a}}$ \\
Proprioceptive & 0.629 & $<0.001^{\mathrm{a}}$ \\
\hline
\end{tabular}

Legend: AQ = Japanese version of Autism Spectrum Quotient; GSQ = Japanese version of

Glasgow Sensory Questionnaire.

${ }^{\mathrm{a}} \mathrm{A}$ significant correlation among the total AQ score was defined as $\mathrm{p} \leq 0.01$. 


\section{Figure legends}

Fig. 1. Correlation between the total GSQ score and AQ score. The total sensory score had a positive linear relationship with the AQ score in the total study population $(r=0.626, p<$ 0.001), in the ASD group $(\mathrm{r}=0.553, \mathrm{p}<0.001)$, and in the control group $(\mathrm{r}=0.559, \mathrm{p}<$ 0.001). AQ = Japanese version of Autism Spectrum Quotient; GSQ = Glasgow Sensory Questionnaire; ASD = autism spectrum disorders 


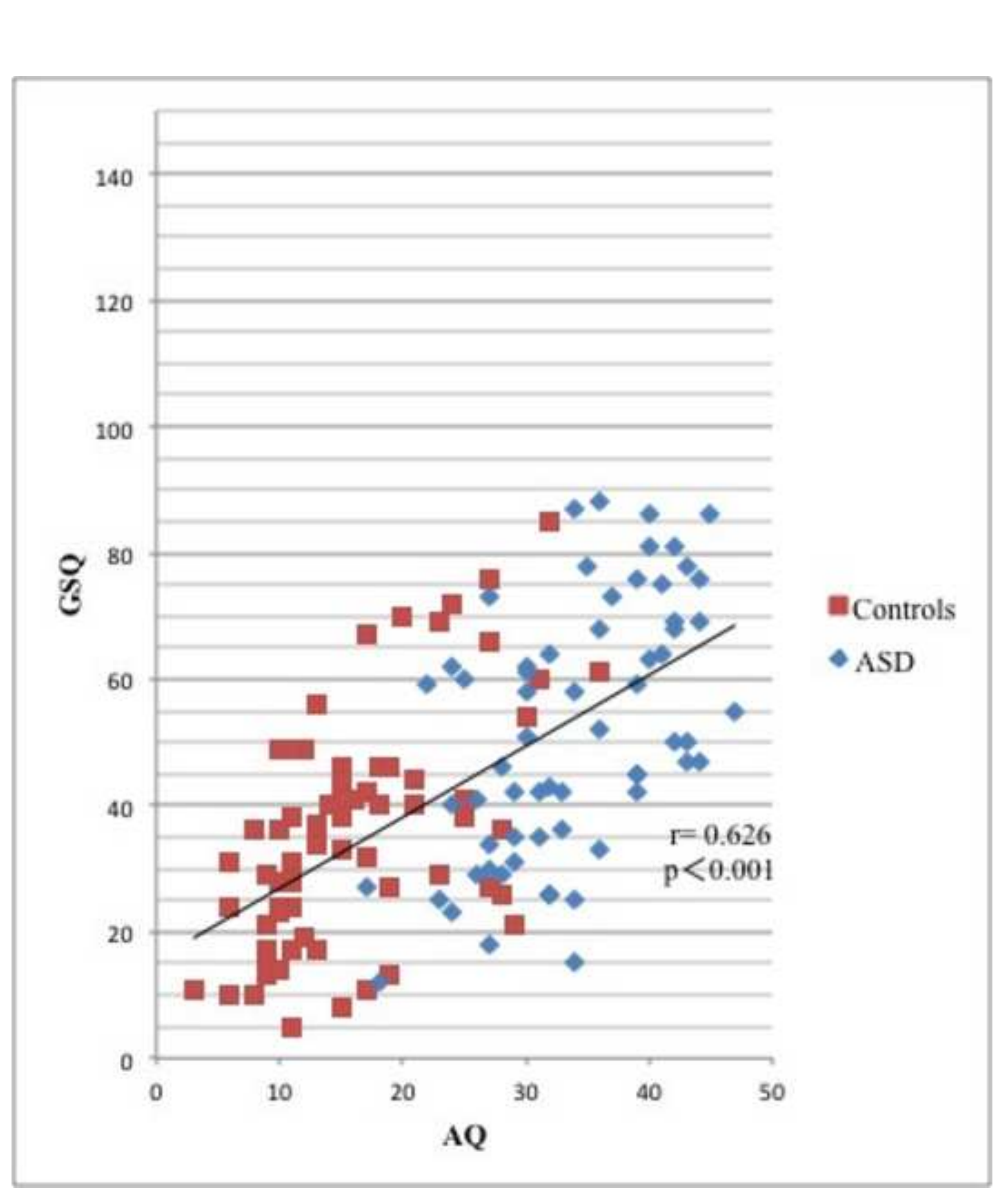

Controls

- ASD

\section{Fure 1}

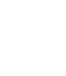

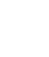

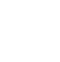

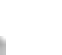

$$
\text { J }
$$
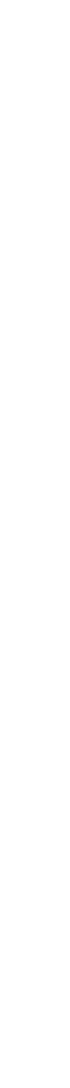